\title{
SOME GENERALIZATIONS OF DARBO FIXED POINT THEOREM AND ITS APPLICATION
}

\begin{abstract}
R. ARAB
Received 14 April, 2014

Abstract. Recently, B. Samet et al. (Fixed Point Theory and Appl. 2013:5, 2013, doi:10.1186/1687-1812-2013-5) has introduced the concept of new contraction mappings and obtained results on the fixed point for nonlinear contractive mappings in a metric space. In the present paper, we introduce the concept of a new contraction via the measure of non-compactness on a Banach space, we obtain a few generalizations of Darbo fixed point theorem and extend some recent results of Aghajani et al. We also show the applicability of obtained results to the theory of integral equations.
\end{abstract}

2010 Mathematics Subject Classification: 54H25; 47H10

Keywords: measure of noncompactness, functional integral equations, fixed point, coupled fixed point

\section{INTRODUCTION}

Integral equation create a very important and significant part of the mathematical analysis and has various applications into real world problems. On the other hand, measures of noncompactness are very useful tools in the wide area of functional analysis such as the metric fixed point theory and the theory of operator equations in Banach spaces. They are also used in the study of functional equations, ordinary and partial differential equations, fractional partial differential equations, integral and integro-differential equations, optimal control theory, etc., see [1-6, 12-16]. In our investigations, we apply the method associated with the technique of measures of noncompactness to generalize the Darbo fixed point theorem [9] and to extend some recent results of Aghajani et al.[5]. Moreover, we are going to study the solvability of the following nonlinear quadratic integral equation of the fractional order

$$
\begin{aligned}
& x(t)=a(t)+h(t,x(t)) \int_{0}^{t} \frac{\left(t^{m}-s^{m}\right)^{\alpha-1}}{\Gamma(\alpha)} m s^{m-1} k_{1}(f(t, s)) x(s) d s \\
& \times \int_{0}^{t} \frac{\left(t^{n}-s^{n}\right)^{\beta-1}}{\Gamma(\beta)} n s^{n-1} k_{2}(g(t, s)) x(s) d s, \\
& \quad t \in I=[0,1], 0<\alpha, \beta \leq 1, m, n>0,
\end{aligned}
$$


where, $\Gamma$ (.) is the (Euler's) Gamma function defined by $\Gamma(\alpha)=\int_{0}^{\infty} t^{\alpha-1} e^{-t} d t$. Let us recall that the function $h(t, x)$ involved in Eq. (1.1) generates the superposition operator $H$ defined by the formula $(H x)(t)=h(t, x(t))$ where $x=x(t)$ is an arbitrary function defined on $I$ (cf.[7],[11]). We show that Eq. (1.1) has solutions in $C(I)$ which are nondecreasing on the interval $I$.

Recently, Samet et al. [17] obtained the following useful generalization of the Banach contraction principle.

Theorem 1. Let $(X, d)$ be a complete metric space, $\varphi: X \rightarrow \mathbb{R}_{+}$be a lower semi continuous function and $T: X \rightarrow X$ be a given mapping. Suppose that for each $0<a<b<\infty$, there exists $0<\gamma(a, b)<1$ such that

$$
\begin{aligned}
& a \leq d(x, y)+\varphi(x)+\varphi(y) \leq b \\
& \quad \Longrightarrow d(T x, T y)+\varphi(T x)+\varphi(T y) \leq \gamma(a, b)[d(x, y)+\varphi(x)+\varphi(y)]
\end{aligned}
$$

for all $x, y \in X$. Then $T$ has a unique fixed point $x \in X$.

\section{AuXiliary FACTS}

In this section, we recall some notations, definitions and theorems to obtain all results of this work. Denote by $\mathbb{R}$ the set of real numbers and put $\mathbb{R}_{+}=[0,+\infty)$. Let $(E,\|\cdot\|)$ be a real Banach space. Let $\bar{B}(x, r)$ denote the closed ball centered at $x$ with radius $r$. The symbol $\bar{B}_{r}$ stands for the ball $\bar{B}(0, r)$. For $X$, a nonempty subset of $E$, we denote by $\bar{X}$ and $\operatorname{Conv} X$ the closure and the convex closure of $X$, respectively. Moreover, let us denote by $\mathfrak{M}_{E}$ the family of nonempty bounded subsets of $E$ and by $\mathfrak{N}_{E}$ its subfamily consisting of all relatively compact sets. We use the following definition of the measure of noncompactness given in [9].

Definition 1. A mapping $\mu: \mathfrak{M}_{E} \rightarrow \mathbb{R}_{+}$is said to be a measure of non-compactness in $E$ if it satisfies the following conditions:

$\left(1^{0}\right)$ : The family $\operatorname{ker} \mu=\left\{X \in \mathfrak{M}_{E}: \mu(X)=0\right\}$ is nonempty and ker $\mu \subset \mathfrak{N}_{E}$,

$\left(2^{0}\right): X \subset Y \Rightarrow \mu(X) \leq \mu(Y)$,

$\left(3^{0}\right): \mu(\bar{X})=\mu(X)$,

(40): $\mu(\operatorname{Conv} X)=\mu(X)$,

$\left(5^{0}\right): \mu(\lambda X+(1-\lambda) Y) \leq \lambda \mu(X)+(1-\lambda) \mu(Y)$ for $\lambda \in[0,1]$,

$\left(6^{0}\right)$ : If $\left(X_{n}\right)$ is a sequence of closed sets from $m_{E}$ such that $X_{n+1} \subset X_{n}(n=$ $1,2, \ldots)$ and if $\lim _{n \longrightarrow \infty} \mu\left(X_{n}\right)=0$, then the set $X_{\infty}=\bigcap_{n=1}^{\infty} X_{n}$ is nonempty.

The family ker $\mu$ defined in axiom $\left(1^{0}\right)$ is called the kernel of the measure of noncompactness $\mu$. One of the properties of the measure of noncompactness is $X_{\infty} \in$ ker $\mu$. Indeed, from the inequality $\mu\left(X_{\infty}\right) \leq \mu\left(X_{n}\right)$ for $n=1,2,3, \ldots$, we infer that 
$\mu\left(X_{\infty}\right)=0$. Further facts concerning measures of noncompactness and their properties may be found in [8,9]. Darbo's fixed point theorem is a very important generalization of Schauder's fixed point theorem, and includes the existence part of Banach's fixed point theorem.

Theorem 2 (Schauder [3]). Let $C$ be a nonempty, bounded, closed, convex subset of a Banach space $E$. Then every compact, continuous map $T: C \rightarrow C$ has at least one fixed point.

In the following we state a fixed-point theorem of Darbo type proved by Banaś and Goebel [9].

Theorem 3. Let $C$ be a nonempty, closed, bounded, and convex subset of the Banach space $E$ and $F: C \rightarrow C$ be a continuous mapping. Assume that there exist a constant $k \in[0,1)$ such that $\mu(F X) \leq k \mu(X)$ for any nonempty subset of $C$. Then $F$ has a fixed-point in $C$.

Remark 1. [9] Under the assumptions of the above theorem it can be shown that the set FixF of fixed points of $F$ belonging to $\Omega$ is an element of ker $\mu$.

\section{FIXED POINT THEOREM}

The main result of the present paper is the following fixed point theorem which is a generalization of Darbo fixed point theorem (cf. Theorem3).

Theorem 4. Let $C$ be a nonempty, bounded, closed and convex subset of a Banach space $E$ and let $\varphi: \mathbb{R}_{+} \longrightarrow \mathbb{R}_{+}$and $T: C \rightarrow C$ be two continuous functions such that for any $0<a<b<\infty$, there exists $0<\gamma(a, b)<1$ such that for all $X \subseteq C$,

$$
a \leq \mu(X)+\varphi(\mu(X)) \leq b \Longrightarrow \mu(T X)+\varphi(\mu(T X)) \leq \gamma(a, b)[\mu(X)+\varphi(\mu(X))],
$$

where $\mu$ is an arbitrary measure of noncompactness. Then $T$ has at least one fixed point in $C$.

Proof. Let $C_{0}=C$, we construct a sequence $\left\{C_{n}\right\}$ such that $C_{n+1}=\operatorname{Conv}\left(T C_{n}\right)$, for $n \geq 0 . T C_{0}=T C \subseteq C=C_{0}, C_{1}=\operatorname{Conv}\left(T C_{0}\right) \subseteq C=C_{0}$, therefore by continuing this process, we have

$$
C_{0} \supseteq C_{1} \supseteq C_{2} \supseteq \ldots \supseteq C_{n} \supseteq C_{n+1} \supseteq \ldots
$$

If there exists a positive integer $N \in \mathbb{N}$ such that $\mu\left(C_{N}\right)+\varphi\left(\mu\left(C_{N}\right)\right)=0$,i.e, $\mu\left(C_{N}\right)=$ 0 , then $C_{N}$ is relatively compact. On the other hand, we have $T\left(C_{N}\right) \subseteq \operatorname{Conv}\left(T C_{N}\right)=$ $C_{N+1} \subseteq C_{N}$. Then Theorem 2 implies that $T$ has a fixed point. So we assume that

$$
0<\mu\left(C_{n}\right)+\varphi\left(\mu\left(C_{n}\right)\right), \quad \forall n \geq 1 .
$$

Suppose that

$$
\mu\left(C_{n_{0}}\right)+\varphi\left(\mu\left(C_{n_{0}}\right)\right)<\mu\left(C_{n_{0}+1}\right)+\varphi\left(\mu\left(C_{n_{0}+1}\right)\right)
$$


for some $n_{0} \in \mathbb{N}$. In addition, by (3.2) and (3.3), we have

$$
\begin{aligned}
0<a:=\mu\left(C_{n_{0}}\right)+\varphi\left(\mu\left(C_{n_{0}}\right)\right) \leq \mu\left(C_{n_{0}}\right) & +\varphi\left(\mu\left(C_{n_{0}}\right)\right) \\
& <\mu\left(C_{n_{0}+1}\right)+\varphi\left(\mu\left(C_{n_{0}}+1\right)\right):=b .
\end{aligned}
$$

By using (3.1), there exists $0<\gamma(a, b)<1$ such that

$$
\begin{aligned}
\mu\left(C_{n_{0}+1}\right)+\varphi\left(\mu\left(C_{n_{0}}+1\right)\right)=\mu(\operatorname{conv} & \left.T C_{n_{0}}\right)+\varphi\left(\mu\left(\operatorname{conv} T C_{n_{0}}\right)\right) \\
=\mu\left(T C_{n_{0}}\right)+\varphi\left(\mu\left(T C_{n_{0}}\right)\right) \leq & \gamma(a, b)\left[\mu\left(C_{n_{0}}\right)+\varphi\left(\mu\left(C_{n_{0}}\right)\right)\right] \\
& <\gamma(a, b)\left[\mu\left(C_{n_{0}+1}\right)+\varphi\left(\mu\left(C_{n_{0}}+1\right)\right)\right],
\end{aligned}
$$

which implies that $\gamma(a, b)>1$, a contradiction. This implies that

$$
\mu\left(C_{n+1}\right)+\varphi(\mu(C n+1)) \leq \mu\left(C_{n}\right)+\varphi\left(\mu\left(C_{n}\right)\right),
$$

for all $n \in \mathbb{N}$, that is, the sequence $\left\{\mu\left(C_{n}\right)+\varphi\left(\mu\left(C_{n}\right)\right)\right\}$ is non-increasing and nonnegative, we infer that

$$
\lim _{n \rightarrow \infty} \mu\left(C_{n}\right)+\varphi\left(\mu\left(C_{n}\right)\right)=\delta .
$$

Now, we show that $\delta=0$. Suppose, to the contrary, that $\delta>0$. Then

$$
0<a:=\delta \leq \mu\left(C_{n}\right)+\varphi\left(\mu\left(C_{n}\right)\right) \leq \mu\left(C_{0}\right)+\varphi\left(\mu\left(C_{0}\right)\right)=: b, \text { for all } n \geq 0 .
$$

By (3.1), there exists $0<\gamma(a, b)<1$ such that

$$
\begin{aligned}
\mu\left(C_{n+1}\right)+\varphi\left(\mu\left(C_{n}+1\right)\right) & =\mu\left(\operatorname{conv} T C_{n}\right)+\varphi\left(\mu\left(\operatorname{conv} T C_{n}\right)\right) \\
& =\mu\left(T C_{n}\right)+\varphi\left(\mu\left(T C_{n}\right)\right) \leq \gamma(a, b)\left[\mu\left(C_{n}\right)+\varphi\left(\mu\left(C_{n}\right)\right)\right] .
\end{aligned}
$$

Letting $n \longrightarrow \infty$ in the above inequality and using (3.4), we get $\delta \leq \gamma(a, b) \delta$, which implies that $\gamma(a, b) \geq 1$, a contradiction. Thus, $\delta=0$ and

$$
\lim _{n \rightarrow \infty} \mu\left(C_{n}\right)+\varphi\left(\mu\left(C_{n}\right)\right)=0 .
$$

It follows that

$$
\lim _{n \rightarrow \infty} \mu\left(C_{n}\right)=0 .
$$

Since $C_{n} \supseteq C_{n+1}$ and $T C_{n} \subseteq C_{n}$ for all $n=1,2, \ldots$, it follows from $\left(6^{0}\right)$ that

$$
C_{\infty}=\bigcap_{n=1}^{\infty} C_{n}
$$

is nonempty convex closed set, invariant under $T$ and belongs to $\operatorname{Ker} \mu$. Therefore Theorem 2 completes the proof.

An immediate consequence of Theorem 4 is the following. 
Theorem 5. Let $C$ be a nonempty, bounded, closed and convex subset of a Banach space E. Let $\varphi: \mathbb{R}_{+} \longrightarrow \mathbb{R}_{+}$and $T: C \rightarrow C$ be continuous functions. Suppose that there exists a constant $0<\lambda<1$, such that

$$
\mu(T X)+\varphi(\mu(T X)) \leq \lambda[\mu(X)+\varphi(\mu(X))],
$$

for all $X \subseteq C$, where $\mu$ is an arbitrary measure of noncompactness. Then $T$ has at least one fixed point in $C$.

Remark 2. Taking $\varphi \equiv 0$ in Theorem 5, we obtain the Darbo fixed point theorem.

Theorem 6. Let $C$ be a nonempty, bounded, closed and convex subset of a Banach space $E$ and let $T: C \rightarrow C$ be a continuous operator satisfying

$$
\psi[\mu(T X)+\varphi(\mu(T X))] \leq \psi[\mu(X)+\varphi(\mu(X))]-\phi[\mu(X)+\varphi(\mu(X))],
$$

for any nonempty $X \subseteq C$, where $\mu$ is an arbitrary measure of noncompactness, $\varphi$ : $\mathbb{R}_{+} \longrightarrow \mathbb{R}_{+}$is continuous function and $\psi, \phi: \mathbb{R}_{+} \longrightarrow \mathbb{R}_{+}$are given functions such that $\psi$ is continuous and nondecreasing function and $\phi$ is lower semicontinuous, $\phi(0)=0$ and $\phi(t)>0$ for all $t>0$. Then $T$ has at least one fixed point in $C$.

Proof. Similarly as in the proof of the Theorem 4, by using induction we define the sequence $\left\{C_{n}\right\}$, where $C_{0}=C$ and $C_{n+1}=\operatorname{Conv}\left(T C_{n}\right)$, for $n \geq 0$. If there exists a positive integer $N_{0} \in \mathbb{N}$ such that $\mu\left(C_{N_{0}}\right)+\varphi\left(\mu\left(C_{N_{0}}\right)\right)=0$, i.e, $\mu\left(C_{N_{0}}\right)=0$, then $C_{N_{0}}$ is relatively compact. On the other hand, $T\left(C_{N_{0}}\right) \subseteq \operatorname{Conv}\left(T C_{N_{0}}\right)=C_{N_{0}+1} \subseteq$ $C_{N_{0}}$, then theorem 2 implies that $T$ has a fixed point. So we assume that

$$
0<\mu\left(C_{n}\right)+\varphi\left(\mu\left(C_{n}\right)\right), \quad \forall n \geq 1 .
$$

From (3.1), we get

$$
\begin{aligned}
\psi\left[\mu\left(C_{n+1}\right)+\varphi\left(\mu\left(C_{n}+1\right)\right)\right] & =\psi\left[\mu\left(\operatorname{conv} T C_{n}\right)+\varphi\left(\mu\left(\operatorname{conv} T C_{n}\right)\right)\right] \\
& =\psi\left[\mu\left(T C_{n}\right)+\varphi\left(\mu\left(T C_{n}\right)\right)\right] \\
& \leq \psi\left[\mu\left(C_{n}\right)+\varphi\left(\mu\left(C_{n}\right)\right)\right]-\phi\left[\mu\left(C_{n}\right)+\varphi\left(\mu\left(C_{n}\right)\right)\right] \\
& \leq \psi\left[\mu\left(C_{n}\right)+\varphi\left(\mu\left(C_{n}\right)\right)\right] .
\end{aligned}
$$

On the other hand, $\psi$ is nondecreasing, then

$$
\mu\left(C_{n+1}\right)+\varphi\left(\mu\left(C_{n}+1\right)\right) \leq \mu\left(C_{n}\right)+\varphi\left(\mu\left(C_{n}\right)\right)
$$

that is, the sequence $\left\{\mu\left(C_{n}\right)+\varphi\left(\mu\left(C_{n}\right)\right)\right\}$ is nonincreasing and nonnegative. We infer that

$$
\lim _{n \rightarrow \infty} \mu\left(C_{n}\right)+\varphi\left(\mu\left(C_{n}\right)\right)=\delta .
$$

Now, we show that $\delta=0$. Suppose, to the contrary, that $\delta>0$. Passing to the (upper) limit in (3.6), when $n \longrightarrow \infty$, it follows that

$$
\psi(\delta) \leq \psi(\delta)-\liminf _{n \longrightarrow \infty} \phi\left[\mu\left(C_{n}\right)+\varphi\left(\mu\left(C_{n}\right)\right)\right] \leq \psi(\delta)-\phi(\delta),
$$


i.e. $\phi(\delta) \leq 0$. Using the properties of $\phi$ function, we get that $\delta=0$ which is a contradiction. We conclude that

$$
\lim _{n \rightarrow \infty} \mu\left(C_{n}\right)+\varphi\left(\mu\left(C_{n}\right)\right)=0,
$$

therefore,

$$
\lim _{n \rightarrow \infty} \mu\left(C_{n}\right)=0 .
$$

Since $C_{n} \supseteq C_{n+1}$ and $T C_{n} \subseteq C_{n}$ for all $n=1,2, \ldots$ It follows from $\left(6^{0}\right)$ that

$$
C_{\infty}=\bigcap_{n=1}^{\infty} C_{n}
$$

is nonempty convex closed set, invariant under $T$ and belongs to $\operatorname{Ker} \mu$. Therefore Theorem 2 completes the proof.

Now, the following fixed point theorem follows immediately from Theorem 6 is a generalization of [5].

Theorem 7. Let $C$ be a nonempty, bounded, closed and convex subset of a Banach space $E$ and let $T: C \rightarrow C$ be continuous operator satisfying

$$
\psi(\mu(T X)) \leq \psi(\mu(X))-\phi(\mu(X)),
$$

for any nonempety $X \subseteq C$, where $\mu$ is an arbitrary measure of noncompactness and $\psi, \phi: \mathbb{R}_{+} \longrightarrow \mathbb{R}_{+}$are given functions such that $\psi$ is continuous and nondecreasing function and $\phi$ is lower semicontinuous, $\phi(0)=0$ and $\phi(t)>0$ for all $t>0$. Then $T$ has at least one fixed point in $C$.

\section{AN APPLICATION TO EXISTENCE OF SOLUTION FOR A FUNCTIONAL INTEGRAL EQUATION.}

Let $C(I)=C[0,1]$ be the Banach space of all continuous functions on $I=[0,1]$ equipped with the standard norm

$$
\|x\|=\max \{|x(t)|: t \geq 0\} .
$$

Next, we recall the definition of a measure of noncompactness in $C(I)$ which will be used in this Section. This measure was introduced and studied in [10]. Let $X$ be a fixed nonempty and bounded subset of $C(I)$. For $x \in X$ and $\epsilon \geq 0$, denote by $\omega(x, \epsilon)$ the modulus of continuity of the function $x$ on the interval $[0,1]$, i.e.

$$
\omega(x, \epsilon):=\sup \{|x(t)-x(s)|: t, s \in[0,1],|t-s| \leq \epsilon\} .
$$

Further, let us put

$$
\omega(X, \epsilon):=\sup \{\omega(x, \epsilon): x \in X\}, \omega_{0}(X):=\lim _{\epsilon \rightarrow 0} \omega(X, \epsilon) .
$$

Define

$$
i(x):=\sup \{|x(s)-x(t)|-[x(s)-x(t)]: t, s \in I, t \leq s\},
$$


and

$$
i(X):=\sup \{i(x): x \in X\} .
$$

Observe that all functions belonging to $X$ are nondecreasing on $I$ if and only if $i(X)=0$.

Now, let us define the function $\mu$ on the family $\mathfrak{M}_{C}(I)$ by the formula

$$
\mu(X):=\omega_{0}(X)+i(X) .
$$

It can be shown [10] that the function $\mu$ is a measure of noncompactness in the space $C(I)$.

Now, equation (1.1) will be investigated under the assumptions:

(i): $a: I \rightarrow \mathbb{R}_{+}$is a continuous, nondecreasing and nonnegative function on $I$;

(ii): $h: I \times \mathbb{R} \rightarrow \mathbb{R}$ is continuous function in $t, x$ such that $h\left(I \times \mathbb{R}_{+}\right) \subseteq \mathbb{R}_{+}$and there exists a nonnegative constant $c$ such that

$$
|h(t, x)-h(t, y)| \leq c|x-y|
$$

for all $t \in I$ and all $x, y \in \mathbb{R}$;

(iii): The superposition operator $H$ generated by the function $h(t, x)$ satisfies for any nonnegative function $x$ the condition $i(H x) \leq c i(x)$ where $c$ is the same constant as in $(i i)$;

(iv): $f, g: I \times I \rightarrow \mathbb{R}$ are continuous and the functions $f(t, s)$ and $g(t, s)$ are nondecreasing for each variable $t$ and $s$, separately;

(v): $k_{1}: \operatorname{Im} f \rightarrow \mathbb{R}_{+}$is a continuous and nondecreasing function on the compact set $\operatorname{Imf}$;

(vi): $k_{2}: \operatorname{Img} \rightarrow \mathbb{R}_{+}$is a continuous and nondecreasing function on the compact set $I m g$;

(vii): The inequality

$$
M_{1} \Gamma(\alpha+1) \Gamma(\beta+1)+\left(c r+M_{2}\right)\left\|k_{1}\right\|\left\|k_{2}\right\| r^{2} \leq \Gamma(\alpha+1) \Gamma(\beta+1) r
$$

has a positive solution $r_{0}$ such that $c\left\|k_{1}\right\|\left\|k_{2}\right\| r_{0}^{2} \leq \Gamma(\alpha+1) \Gamma(\beta+1)$, where $M_{1}=\max \{|a(t)|: t \in I\}$ and $M_{2}=\max \{|h(t, 0)|: t \in I\}$.

Theorem 8. Under assumptions ( $i)$ - (vii), the equation (1.1) has at least one solution $x=x(t)$ which belongs to the space $C(I)$ and is nondecreasing on the interval $I$.

Proof. Consider the operators $F, G$ and $T$ defined on the space $C(I)$ by the formulas

$$
\begin{aligned}
& (F x)(t)=\int_{0}^{t} \frac{\left(t^{m}-s^{m}\right)^{\alpha-1}}{\Gamma(\alpha)} m s^{m-1} k_{1}(f(t, s)) x(s) d s, \\
& (G x)(t)=\int_{0}^{t} \frac{\left(t^{n}-s^{n}\right)^{\beta-1}}{\Gamma(\beta)} n s^{n-1} k_{2}(g(t, s)) x(s) d s, \\
& (T x)(t)=a(t)+h(t, x(t))(F x)(t)(G x)(t) .
\end{aligned}
$$


Firstly, we prove that $F$ and $G$ are selfmaps on $C(I)$. To this end, it suffices to show that if $x \in C(I)$ then $F x, G x \in C(I)$. Fix $\epsilon>0$, let $x \in C(I)$ and let $t_{1}, t_{2} \in I$ (without loss of generality assume that $t_{2} \geq t_{1}$ ) and $\left|t_{2}-t_{1}\right| \leq \epsilon$. Then we get

$$
\begin{gathered}
\left|(F x)\left(t_{2}\right)-(F x)\left(t_{1}\right)\right| \leq \mid \int_{0}^{t_{2}} \frac{\left(t_{2}^{m}-s^{m}\right)^{\alpha-1}}{\Gamma(\alpha)} m s^{m-1} k_{1}\left(f\left(t_{2}, s\right)\right) x(s) d s \\
-\int_{0}^{t_{2}} \frac{\left(t_{2}^{m}-s^{m}\right)^{\alpha-1}}{\Gamma(\alpha)} m s^{m-1} k_{1}\left(f\left(t_{1}, s\right)\right) x(s) d s \mid \\
+\mid \int_{0}^{t_{2}} \frac{\left(t_{2}^{m}-s^{m}\right)^{\alpha-1}}{\Gamma(\alpha)} m s^{m-1} k_{1}\left(f\left(t_{1}, s\right)\right) x(s) d s \\
-\int_{0}^{t_{1}} \frac{\left(t_{2}^{m}-s^{m}\right)^{\alpha-1}}{\Gamma(\alpha)} m s^{m-1} k_{1}\left(f\left(t_{1}, s\right)\right) x(s) d s \mid \\
+\mid \int_{0}^{t_{1}} \frac{\left(t_{2}^{m}-s^{m}\right)^{\alpha-1}}{\Gamma(\alpha)} m s^{m-1} k_{1}\left(f\left(t_{1}, s\right)\right) x(s) d s \\
-\int_{0}^{t_{1}} \frac{\left(t_{1}^{m}-s^{m}\right)^{\alpha-1}}{\Gamma(\alpha)} m s^{m-1} k_{1}\left(f\left(t_{1}, s\right)\right) x(s) d s \mid \\
\leq \int_{0}^{t_{2}} \frac{\left(t_{2}^{m}-s^{m}\right)^{\alpha-1} m s^{m-1}\left|k_{1}\left(f\left(t_{2}, s\right)\right)-k_{1}\left(f\left(t_{1}, s\right)\right)\right||x(s)| d s}{\Gamma(\alpha)} \\
+\int_{t_{1}}^{t_{2}} \frac{\left(t_{2}^{m}-s^{m}\right)^{\alpha-1}}{\Gamma(\alpha)} m s^{m-1}\left|k_{1}\left(f\left(t_{1}, s\right)\right)\right||x(s)| d s \\
+\int_{0}^{t_{1}} \frac{\left|\left(t_{2}^{m}-s^{m}\right)^{\alpha-1}-\left(t_{1}^{m}-s^{m}\right)^{\alpha-1}\right|}{\Gamma(\alpha)} m s^{m-1}\left|k_{1}\left(f\left(t_{1}, s\right)\right)\right||x(s)| d s .
\end{gathered}
$$

Therefore, if we denote

$$
\omega_{k_{1} o f}(\epsilon, .)=\sup \left\{\left|k_{1}(f(t, s))-k_{1}\left(f\left(t^{\prime}, s\right)\right)\right|: t, t^{\prime}, s \in I \text { and }\left|t-t^{\prime}\right| \leq \epsilon\right\}
$$

then

$$
\begin{gathered}
\left|(F x)\left(t_{2}\right)-(F x)\left(t_{1}\right)\right| \leq \frac{\|x\| \omega_{k_{1} o f}(\epsilon, .)}{\Gamma(\alpha)} \frac{t_{2}^{m \alpha}}{\alpha}+\frac{\|x\|\left\|k_{1}\right\|}{\Gamma(\alpha)} \frac{\left(t_{2}^{m}-t_{1}^{m}\right)^{\alpha}}{\alpha} \\
+\frac{\|x\|\left\|k_{1}\right\|}{\Gamma(\alpha)}\left[\frac{\left(t_{2}^{m}-t_{1}^{m}\right)^{\alpha}}{\alpha}+\frac{t_{1}^{m \alpha}}{\alpha}-\frac{t_{2}^{m \alpha}}{\alpha}\right] \\
\quad \leq \frac{\|x\| \omega_{k_{1} o f}(\epsilon, .)}{\Gamma(\alpha+1)}+\frac{2\|x\|\left\|k_{1}\right\|}{\Gamma(\alpha+1)}\left(t_{2}^{m}-t_{1}^{m}\right)^{\alpha} .
\end{gathered}
$$

Similarly, one can show that

$$
\left|(G x)\left(t_{2}\right)-(G x)\left(t_{1}\right)\right| \leq \frac{\|x\| \omega_{k_{2} o g}(\epsilon, .)}{\Gamma(\beta+1)}+\frac{2\|x\|\left\|k_{2}\right\|}{\Gamma(\beta+1)}\left(t_{2}^{n}-t_{1}^{n}\right)^{\beta},
$$


where

$$
\omega_{k_{2} o g}(\epsilon, .)=\sup \left\{\left|k_{2}(g(t, s))-k_{2}\left(g\left(t^{\prime}, s\right)\right)\right|: t, t^{\prime}, s \in I \text { and }\left|t-t^{\prime}\right| \leq \epsilon\right\} .
$$

Obviously, $\omega_{k_{1} \text { of }}(\epsilon,.) \longrightarrow 0$ as $\epsilon \longrightarrow 0$ which is a simple consequence of the uniform continuity of the function $k_{1}$ of on the set $I \times I$. Thus $F x \in C(I)$, and similar to the previous case $G x \in C(I)$, and consequently, $T x \in C(I)$. Also, we have

$$
|(F x)(t)| \leq \int_{0}^{t} \frac{\left(t^{m}-s^{m}\right)^{\alpha-1}}{\Gamma(\alpha)} m s^{m-1}\left|k_{1}(f(t, s))\right||x(s)| d s \leq \frac{\left\|k_{1}|\||| x\|\right.}{\Gamma(\alpha+1)}
$$

for all $t \in I$. On the other hand,

$$
|(G x)(t)| \leq \int_{0}^{t} \frac{\left(t^{n}-s^{n}\right)^{\beta-1}}{\Gamma(\beta)} n s^{n-1}\left|k_{2}(g(t, s))\right||x(s)| d s \leq \frac{\left\|k_{2}|||| x\right\|}{\Gamma(\beta+1)} .
$$

Linking (4.3) and (4.4) we obtain

$$
\begin{aligned}
|(T x)(t)| \leq & |a(t)|+|h(t, x) \| F x(t)||G x(t)| \\
\leq M_{1}+[|h(t, x)-h(t, 0)| & +|h(t, 0)|] \frac{\left\|k_{1}\right\|\left\|k_{2}\right\|\|x\|^{2}}{\Gamma(\alpha+1) \Gamma(\beta+1)} \\
& \leq M_{1}+\left(c\|x\|+M_{2}\right) \frac{\left\|k_{1}\right\|\left\|k_{2}\right\|\|x\|^{2}}{\Gamma(\alpha+1) \Gamma(\beta+1)} .
\end{aligned}
$$

Hence,

$$
\|T x\| \leq M_{1}+\left(c\|x\|+M_{2}\right) \frac{\left\|k_{1}\right\|\left\|k_{2}\right\|\|x\|^{2}}{\Gamma(\alpha+1) \Gamma(\beta+1)} .
$$

Thus, if $\|x\| \leq r_{0}$ we obtain from assumption (vii) the estimate

$$
\|T x\| \leq M_{1}+\left(c r_{0}+M_{2}\right) \frac{\left\|k_{1}\right\|\left\|k_{2}\right\| r_{0}^{2}}{\Gamma(\alpha+1) \Gamma(\beta+1)} \leq r_{0} .
$$

Consequently, the operator $T$ maps the ball $B_{r_{0}} \subset C(I)$ into itself. Next, we prove that the operator $T$ is continuous on $B_{r_{0}}$. To do this, let $\left\{x_{n}\right\}$ be a sequence in $B_{r_{0}}$ such that $x_{n} \rightarrow x$. We have to show that $T x_{n} \rightarrow T x$. In fact, for each $t \in I$, we have

$$
\begin{aligned}
\left|\left(F x_{n}\right)(t)-(F x)(t)\right| & =\mid \int_{0}^{t} \frac{\left(t^{m}-s^{m}\right)^{\alpha-1}}{\Gamma(\alpha)} m s^{m-1} k_{1}(f(t, s)) x_{n}(s) d s \\
-\int_{0}^{t} & \frac{\left(t^{m}-s^{m}\right)^{\alpha-1}}{\Gamma(\alpha)} m s^{m-1} k_{1}(f(t, s)) x(s) d s \mid \\
\leq & \int_{0}^{t} \frac{\left(t^{m}-s^{m}\right)^{\alpha-1}}{\Gamma(\alpha)} m s^{m-1}\left|k_{1}(f(t, s))\right|\left|x_{n}(s)-x(s)\right| d s,
\end{aligned}
$$

thus

$$
\left\|F x_{n}-F x\right\| \leq \frac{\left\|k_{1}\right\|}{\Gamma(\alpha+1)}\left\|x_{n}-x\right\| .
$$


Similarly, we have

$$
\left\|G x_{n}-G x\right\| \leq \frac{\left\|k_{2}\right\|}{\Gamma(\beta+1)}\left\|x_{n}-x\right\|
$$

As,

$$
\begin{aligned}
& \left|\left(T x_{n}\right)(t)-(T x)(t)\right|= \\
& =\left|h\left(t, x_{n}(t)\right)\left(F x_{n}\right)(t)\left(G x_{n}\right)(t)-h(t, x(t))(F x)(t)(G x)(t)\right| \\
& \leq\left|h\left(t, x_{n}(t)\right)\left(F x_{n}\right)(t)\left(G x_{n}\right)(t)-h(t, x(t))\left(F x_{n}\right)(t)\left(G x_{n}\right)(t)\right| \\
& \quad+\left|h(t, x(t))\left(F x_{n}\right)(t)\left(G x_{n}\right)(t)-h(t, x(t))(F x)(t)\left(G x_{n}\right)(t)\right| \\
& \quad+\left|h(t, x(t))(F x)(t)\left(G x_{n}\right)(t)-h(t, x(t))(F x)(t)(G x)(t)\right| \\
& \leq\left|h\left(t, x_{n}(t)\right)-h(t, x(t))\right|\left|\left(F x_{n}\right)(t)\right|\left|\left(G x_{n}\right)(t)\right| \\
& \quad+|h(t, x(t))|\left|\left(F x_{n}\right)(t)-(F x)(t)\right|\left|\left(G x_{n}\right)(t)\right| \\
& \quad+|h(t, x(t))||(F x)(t)|\left|\left(G x_{n}\right)(t)-(G x)(t)\right| \\
& \leq c\left|x_{n}(t)-x(t)\right| \int_{0}^{t} \frac{\left(t^{m}-s^{m}\right)^{\alpha-1}}{\Gamma(\alpha)} m s^{m-1}\left|k_{1}(f(t, s))\right|\left|x_{n}(s)\right| d s \\
& \quad \times \int_{0}^{t} \frac{\left(t^{n}-s^{n}\right)^{\beta-1}}{\Gamma(\beta)} n s^{n-1}\left|k_{2}(g(t, s))\right|\left|x_{n}(s)\right| d s \\
& \quad+\left(c|x(t)|+M_{2}\right) \int_{0}^{t} \frac{\left(t^{m}-s^{m}\right)^{\alpha-1}}{\Gamma(\alpha)} m s^{m-1}\left|k_{1}(f(t, s))\right|\left|x_{n}(s)-x(s)\right| d s \\
& \quad \times \int_{0}^{t} \frac{\left(t^{n}-s^{n}\right)^{\beta-1}}{\Gamma(\beta)} n s^{n-1}\left|k_{2}(g(t, s))\right|\left|x_{n}(s)\right| d s \\
& \quad+\left(c|x(t)|+M_{2}\right) \int_{0}^{t} \frac{\left(t^{m}-s^{m}\right)^{\alpha-1}}{\Gamma(\alpha)} m s^{m-1}\left|k_{1}(f(t, s))\right||x(s)| d s \\
& \quad \times \int_{0}^{t} \frac{\left(t^{n}-s^{n}\right)^{\beta-1}}{\Gamma(\beta)} n s^{n-1}\left|k_{2}(g(t, s))\right|\left|x_{n}(s)-x(s)\right| d s .
\end{aligned}
$$

It follows that

$$
\begin{aligned}
\| T x_{n}-T x \mid \leq & c\left\|x_{n}-x\right\| \frac{\left\|k_{1}\right\|}{\Gamma(\alpha+1)} \frac{\left\|k_{2}\right\|}{\Gamma(\beta+1)}\left\|x_{n}\right\|^{2} \\
& +\left(c\|x\|+M_{2}\right) \frac{\left\|k_{1}\right\|}{\Gamma(\alpha+1)}\left\|x_{n}-x\right\| \frac{\left\|k_{2}\right\|}{\Gamma(\beta+1)}\left\|x_{n}\right\| \\
& +\left(c\|x\|+M_{2}\right) \frac{\left\|k_{1}\right\|}{\Gamma(\alpha+1)}\|x\| \frac{\left\|k_{2}\right\|}{\Gamma(\beta+1)}\left\|x_{n}-x\right\| .
\end{aligned}
$$

This proves that $T$ is continuous on $B_{r_{0}}$. Consider the operator $T$ on the subset $B_{r_{0}}^{+}$ of the ball $B_{r_{0}}$ defined in the following way:

$$
B_{r_{0}}^{+}=\left\{x \in B_{r_{0}}: x(t) \geq 0, \text { for } t \in I\right\} .
$$


Obviously, the set $B_{r_{0}}^{+}$is nonempty, bounded, closed and convex. In view of our assumptions $(i),(i i i),(v)$ and $(v i)$ if $x(t) \geq 0$ then $(T x)(t) \geq 0$ for all $t \in I$. Thus $T$ transforms the set $B_{r_{0}}^{+}$into itself. Moreover, $T$ is continuous on $B_{r_{0}}^{+}$. Let $X$ be a nonempty subset of $B_{r_{0}}^{+}$. Fix $\epsilon>0$ and $t_{1}, t_{2} \in I$ with $\left|t_{2}-t_{1}\right| \leq \epsilon$. Without loss of generality assume that $t_{2} \geq t_{1}$. Then we get

$$
\begin{aligned}
& \left|(T x)\left(t_{2}\right)-(T x)\left(t_{1}\right)\right|=\mid a\left(t_{2}\right)+h\left(t_{2}, x\left(t_{2}\right)\right)(F x)\left(t_{2}\right)(G x)\left(t_{2}\right) \\
& -a\left(t_{1}\right)-h\left(t_{1}, x\left(t_{1}\right)\right)(F x)\left(t_{1}\right)(G x)\left(t_{1}\right) \\
& \leq\left|a\left(t_{2}\right)-a\left(t_{1}\right)\right|+\mid h\left(t_{2}, x\left(t_{2}\right)\right)(F x)\left(t_{2}\right)(G x)\left(t_{2}\right) \\
& -h\left(t_{1}, x\left(t_{2}\right)\right)(F x)\left(t_{2}\right)(G x)\left(t_{2}\right) \\
& +\left|h\left(t_{1}, x\left(t_{2}\right)\right)(F x)\left(t_{2}\right)(G x)\left(t_{2}\right)-h\left(t_{1}, x\left(t_{1}\right)\right)(F x)\left(t_{2}\right)(G x)\left(t_{2}\right)\right| \\
& +\left|h\left(t_{1}, x\left(t_{1}\right)\right)(F x)\left(t_{2}\right)(G x)\left(t_{2}\right)-h\left(t_{1}, x\left(t_{1}\right)\right)(F x)\left(t_{1}\right)(G x)\left(t_{2}\right)\right| \\
& +\left|h\left(t_{1}, x\left(t_{1}\right)\right)(F x)\left(t_{1}\right)(G x)\left(t_{2}\right)-h\left(t_{1}, x\left(t_{1}\right)\right)(F x)\left(t_{1}\right)(G x)\left(t_{1}\right)\right| \\
& \leq\left|a\left(t_{2}\right)-a\left(t_{1}\right)\right|+\left|h\left(t_{2}, x\left(t_{2}\right)\right)-h\left(t_{1}, x\left(t_{2}\right)\right)\right|\left|(F x)\left(t_{2}\right)\right|\left|(G x)\left(t_{2}\right)\right| \\
& +\left|h\left(t_{1}, x\left(t_{2}\right)\right)-h\left(t_{1}, x\left(t_{1}\right)\right)\right|\left|(F x)\left(t_{2}\right)\right|\left|(G x)\left(t_{2}\right)\right| \\
& +\left|h\left(t_{1}, x\left(t_{1}\right)\right)\right|\left|(F x)\left(t_{2}\right)-(F x)\left(t_{1}\right)\right|\left|(G x)\left(t_{2}\right)\right| \\
& +\left|h\left(t_{1}, x\left(t_{1}\right)\right)\right|\left|(F x)\left(t_{1}\right)\right|\left|(G x)\left(t_{2}\right)-(G x)\left(t_{1}\right)\right| \\
& \leq \omega(a, \epsilon)+\gamma_{r_{0}}(h, \epsilon) \frac{\| x|||| k_{1}||}{\Gamma(\alpha+1)} \frac{\left\|x|||| k_{2}\right\|}{\Gamma(\beta+1)}+c\left|x\left(t_{2}\right)-x\left(t_{1}\right)\right| \frac{\| x|||| k_{1}||}{\Gamma(\alpha+1)} \frac{\left\|x|||| k_{2}\right\|}{\Gamma(\beta+1)} \\
& +\left(c\|x\|+M_{2}\right)\left[\frac{\|x\| \omega_{k_{1} o f}(\epsilon, .)}{\Gamma(\alpha+1)}+\frac{2\|x\| \mid\left\|k_{1}\right\|}{\Gamma(\alpha+1)}\left(t_{2}^{m}-t_{1}^{m}\right)^{\alpha}\right] \frac{\|x\||| \mid k_{2} \|}{\Gamma(\beta+1)} \\
& +\left(c\|x\|+M_{2}\right) \frac{\|x\|\left\|k_{1}\right\|}{\Gamma(\alpha+1)}\left[\frac{\|x\| \omega_{k_{2} o g}(\epsilon, .)}{\Gamma(\beta+1)}+\frac{2\|x\|\left\|k_{2}\right\|}{\Gamma(\beta+1)}\left(t_{2}^{n}-t_{1}^{n}\right)^{\beta}\right],
\end{aligned}
$$

where we denoted

$$
\gamma_{r_{0}}(h, \epsilon)=\sup \left\{\left|h(t, x)-h\left(t^{\prime}, x\right)\right|: t, t^{\prime} \in I, x \in\left[0, r_{0}\right],\left|t-t^{\prime}\right| \leq \epsilon\right\} .
$$

By applying the mean value theorem on $\left[t_{1}, t_{2}\right]$, we get

$$
\left|t_{2}^{m}-t_{1}^{m}\right|^{\alpha} \leq m^{\alpha}\left|t_{2}-t_{1}\right|^{\alpha},
$$

and

$$
\left|t_{2}^{n}-t_{1}^{n}\right|^{\beta} \leq n^{\beta}\left|t_{2}-t_{1}\right|^{\beta}
$$


Thus from the last inequality, we get

$$
\begin{aligned}
\mid(T x)\left(t_{2}\right) & -(T x)\left(t_{1}\right) \mid \leq \omega(a, \epsilon)+\gamma_{r_{0}}(h, \epsilon) \frac{\left\|x \left|\left\||| k_{1}\right\|\right.\right.}{\Gamma(\alpha+1)} \frac{\|x\||| k_{2} \|}{\Gamma(\beta+1)} \\
& +c\left|x\left(t_{2}\right)-x\left(t_{1}\right)\right| \frac{\left\|x \left|\left\||| k_{1}\right\|\right.\right.}{\Gamma(\alpha+1)} \frac{\|x\|\left\||| k_{2}\right\|}{\Gamma(\beta+1)} \\
& +\left(c\|x\|+M_{2}\right)\left[\frac{\|x\| \omega_{k_{1} o f}(\epsilon, .)}{\Gamma(\alpha+1)}+\frac{2\left\|x \left|\left\||| k_{1}\right\|\right.\right.}{\Gamma(\alpha+1)}(m \epsilon)^{\alpha}\right] \frac{\|x\|\left\|k_{2}\right\|}{\Gamma(\beta+1)} \\
& +\left(c\|x\|+M_{2}\right) \frac{\|x\|\left\|k_{1}\right\|}{\Gamma(\alpha+1)}\left[\frac{\|x\| \omega_{k_{2} o g}(\epsilon, .)}{\Gamma(\beta+1)}+\frac{2\|x\|\left\|k_{2}\right\|}{\Gamma(\beta+1)}(n \epsilon)^{\beta}\right],
\end{aligned}
$$

hence,

$$
\begin{aligned}
& \omega(T x, \epsilon) \leq \omega(a, \epsilon)+ \\
&+\left(c r_{0}+M_{2}\right)\left[\frac{r_{0} \omega_{k_{1} o f}(\epsilon, .)}{\Gamma(\alpha+1)}+\frac{r_{0}^{2}\left\|k_{1}\right\|\left\|k_{2}\right\|}{\Gamma(\alpha+1)}+c \omega(x, \epsilon) \mid \frac{r_{0}^{2}\left\|k_{1}\right\|\left\|k_{2}\right\|}{\Gamma(\alpha+1) \Gamma(\beta+1)}(m)^{\alpha}\right] \frac{r_{0}\left\|k_{2}\right\|}{\Gamma(\beta+1)} \\
&+\left(c r_{0}+M_{2}\right) \frac{r_{0}\left\|k_{1}\right\|}{\Gamma(\alpha+1)}\left[\frac{r_{0} \omega_{k_{2} o g}(\epsilon, .)}{\Gamma(\beta+1)}+\frac{2 r_{0}\left\|k_{2}\right\|}{\Gamma(\beta+1)}(n \epsilon)^{\beta}\right] .
\end{aligned}
$$

Thus, taking the supremum on $X$, we obtain

$$
\begin{aligned}
\omega(T X, \epsilon) \leq & \omega(a, \epsilon)+\gamma_{r_{0}}(h, \epsilon) \frac{r_{0}^{2}\left\|k_{1}\right\||| k_{2} \|}{\Gamma(\alpha+1) \Gamma(\beta+1)}+c \omega(X, \epsilon) \mid \frac{r_{0}^{2}\left\|k_{1}\right\|\left\|k_{2}\right\|}{\Gamma(\alpha+1) \Gamma(\beta+1)} \\
& +\left(c r_{0}+M_{2}\right)\left[\frac{r_{0} \omega_{k_{1} o f}(\epsilon, .)}{\Gamma(\alpha+1)}+\frac{2 r_{0}\left\|k_{1}\right\|}{\Gamma(\alpha+1)}(m \epsilon)^{\alpha}\right] \frac{r_{0}\left\|k_{2}\right\|}{\Gamma(\beta+1)} \\
& +\left(c r_{0}+M_{2}\right) \frac{r_{0}\left\|k_{1}\right\|}{\Gamma(\alpha+1)}\left[\frac{r_{0} \omega_{k_{2} o g}(\epsilon, .)}{\Gamma(\beta+1)}+\frac{2 r_{0}\left\|k_{2}\right\|}{\Gamma(\beta+1)}(n \epsilon)^{\beta}\right] .
\end{aligned}
$$

From the uniform continuity of the functions $k_{1} o f$ and $k_{2} o g$ on the set $I \times I$ and $h$ on the set $I \times\left[0, r_{0}\right]$ and the continuity of the function $a$ on $I$, we have that $\omega_{k_{1} o f}(\epsilon,.) \rightarrow$ $0, \omega_{k_{2} o g}(\epsilon,.) \rightarrow 0, \gamma_{r_{0}}(h, \epsilon) \rightarrow 0$ and $\omega(a, \epsilon) \rightarrow 0$ as $\epsilon \rightarrow 0$. So, let $\epsilon \rightarrow 0$ to obtain

$$
\omega_{0}(T X) \leq c \frac{r_{0}^{2}\left\|k_{1}\right\|\left\|k_{2}\right\|}{\Gamma(\alpha+1) \Gamma(\beta+1)} \omega_{0}(X) .
$$


Let $x \in X$ and $t_{1}, t_{2} \in I$ with $t_{1}<t_{2}$. Then

$$
\begin{aligned}
&\left|(T x)\left(t_{2}\right)-(T x)\left(t_{1}\right)\right|-\left[(T x)\left(t_{2}\right)-(T x)\left(t_{1}\right)\right]= \\
&= \mid a\left(t_{2}\right)+h\left(t_{2}, x\left(t_{2}\right)\right)(F x)\left(t_{2}\right)(G x)\left(t_{2}\right) \\
&-a\left(t_{1}\right)-h\left(t_{1}, x\left(t_{1}\right)\right)(F x)\left(t_{1}\right)(G x)\left(t_{1}\right) \mid \\
&-\left[a\left(t_{2}\right)+h\left(t_{2}, x\left(t_{2}\right)\right)(F x)\left(t_{2}\right)(G x)\left(t_{2}\right)\right. \\
&\left.-a\left(t_{1}\right)-h\left(t_{1}, x\left(t_{1}\right)\right)(F x)\left(t_{1}\right)(G x)\left(t_{1}\right)\right] \\
& \leq\left\{\left|a\left(t_{2}\right)-a\left(t_{1}\right)\right|-\left[a\left(t_{2}\right)-a\left(t_{1}\right)\right]\right\}+\mid h\left(t_{2}, x\left(t_{2}\right)\right)(F x)\left(t_{2}\right)(G x)\left(t_{2}\right) \\
&-h\left(t_{1}, x\left(t_{1}\right)\right)(F x)\left(t_{2}\right)(G x)\left(t_{2}\right) \mid \\
&+\left|h\left(t_{1}, x\left(t_{1}\right)\right)(F x)\left(t_{2}\right)(G x)\left(t_{2}\right)-h\left(t_{1}, x\left(t_{1}\right)\right)(F x)\left(t_{1}\right)(G x)\left(t_{2}\right)\right| \\
&+\left|h\left(t_{1}, x\left(t_{1}\right)\right)(F x)\left(t_{1}\right)(G x)\left(t_{2}\right)-h\left(t_{1}, x\left(t_{1}\right)\right)(F x)\left(t_{1}\right)(G x)\left(t_{1}\right)\right| \\
&-\left\{\left[h\left(t_{2}, x\left(t_{2}\right)\right)(F x)\left(t_{2}\right)(G x)\left(t_{2}\right)-h\left(t_{1}, x\left(t_{1}\right)\right)(F x)\left(t_{2}\right)(G x)\left(t_{2}\right)\right]\right. \\
&+\left[h\left(t_{1}, x\left(t_{1}\right)\right)(F x)\left(t_{2}\right)(G x)\left(t_{2}\right)-h\left(t_{1}, x\left(t_{1}\right)\right)(F x)\left(t_{1}\right)(G x)\left(t_{2}\right)\right] \\
&\left.+\left[h\left(t_{1}, x\left(t_{1}\right)\right)(F x)\left(t_{1}\right)(G x)\left(t_{2}\right)-h\left(t_{1}, x\left(t_{1}\right)\right)(F x)\left(t_{1}\right)(G x)\left(t_{1}\right)\right]\right\} \\
& \leq\left\{\left|h\left(t_{2}, x\left(t_{2}\right)\right)-h\left(t_{1}, x\left(t_{1}\right)\right)\right|-\left[h\left(t_{2}, x\left(t_{2}\right)\right)-h\left(t_{1}, x\left(t_{1}\right)\right)\right]\right\}(F x)\left(t_{2}\right)(G x)\left(t_{2}\right) \\
&+h\left(t_{1}, x\left(t_{1}\right)\right)\left\{\left|(F x)\left(t_{2}\right)-(F x)\left(t_{1}\right)\right|-\left[(F x)\left(t_{2}\right)-(F x)\left(t_{1}\right)\right]\right\}(G x)\left(t_{2}\right) \\
&+h\left(t_{1}, x\left(t_{1}\right)\right)(F x)\left(t_{1}\right)\left\{\left|(G x)\left(t_{2}\right)-(G x)\left(t_{1}\right)\right|-\left[(G x)\left(t_{2}\right)-(G x)\left(t_{1}\right)\right]\right\} \\
& \leq i(H x) \frac{r_{0}|| k_{1}||}{\Gamma(\alpha+1)} \frac{r_{0}|| k_{2}||}{\Gamma(\beta+1)} .
\end{aligned}
$$

The above estimate implies that

$$
i(T x) \leq c i(x) \frac{r_{0}^{2}\left\|k _ { 1 } \left|\left\|\mid k_{2}\right\|\right.\right.}{\Gamma(\alpha+1) \Gamma(\beta+1)},
$$

and consequently,

$$
i(T X) \leq \frac{c r_{0}^{2}\left\|k_{1}\right\|\left\|k_{2}\right\|}{\Gamma(\alpha+1) \Gamma(\beta+1)} i(X) .
$$

From (4.5) and (4.6) and the definition of the measure of noncompactness $\mu$, we obtain

$$
\begin{aligned}
\mu(T X) & =\omega_{0}(T X)+i(T X) \\
& \leq c \frac{r_{0}^{2}\left\|k_{1}\right\|\left\|k_{2}\right\|}{\Gamma(\alpha+1) \Gamma(\beta+1)} \omega_{0}(X)+\frac{c r_{0}^{2}\left\|k_{1}\right\|\left\|k_{2}\right\|}{\Gamma(\alpha+1) \Gamma(\beta+1)} i(X) \\
& \leq c \frac{r_{0}^{2}\left\|k_{1}\right\|\left\|\mid k_{2}\right\|}{\Gamma(\alpha+1) \Gamma(\beta+1)}\left(\omega_{0}(X)+i(X)\right) \leq c \frac{r_{0}^{2}\left\|k_{1}\right\|\left\|k_{2}\right\|}{\Gamma(\alpha+1) \Gamma(\beta+1)} \mu(X) .
\end{aligned}
$$

Now, taking into account the above inequality and the fact that $c \frac{r_{0}^{2}\left\|k_{1}\left|\left\|\mid k_{2}\right\|\right.\right.}{\Gamma(\alpha+1) \Gamma(\beta+1)}<1$ and applying Remark 2, we complete the proof. Also, such 
a solution is nondecreasing in view of Remark 1 and the definition of the measure of noncompactness $\mu$ given in Section 2.

In what follows we illustrate the above obtained result by the following example.

Example 1. Consider the following functional integral equation of fractional order

$$
\begin{array}{r}
x(t)=\frac{1}{5} t^{3}+\frac{2 t x(t)}{5(1+t)} \int_{0}^{t} \frac{2 s}{\Gamma\left(\frac{1}{2}\right) \sqrt{t^{2}-s^{2}}}\left[\frac{1}{8}(t+s)+\frac{1}{4}\right] x(s) d s \times \\
\int_{0}^{t} \frac{3 s^{2}}{\Gamma\left(\frac{1}{3}\right) \sqrt[3]{\left(t^{3}-s^{3}\right)^{2}}} \frac{(t+\sqrt{s})^{2}}{12} x(s) d s,
\end{array}
$$

where $t \in I$. In this example, we have $a(t)=\frac{1}{5} t^{3}$ and this function satisfies assumption $(i)$ and $M_{1}=\frac{1}{5}$. Here $f(t, s)=\frac{1}{4} \sqrt{t+s}$ and $g(t, s)=t+\sqrt{s}$ and these functions satisfies assumption (iv). Let $k_{1}:\left[0, \frac{\sqrt{2}}{4}\right] \rightarrow \mathbb{R}_{+}$and $k_{2}:[0,2] \rightarrow \mathbb{R}_{+}$be given by $k_{1}(y)=2 y^{2}+\frac{1}{4}$ and $k_{2}(y)=\frac{1}{12} y^{2}$, then $k_{1}$ and $k_{2}$ satisfying assumptions $(v)$ and $(v i)$ with $\left\|k_{1}\right\|=\frac{1}{2}$ and $\left\|k_{2}\right\|=\frac{1}{3}$. Moreover, the function $h(t, x)=\frac{2 t x}{5(1+t)}$ satisfies hypothesis $(i i)$ and

$$
|h(t, x)-h(t, y)| \leq \frac{1}{5}|x-y|
$$

for all $x, y \in \mathbb{R}$ and $t \in I$. Moreover, the function $h$ satisfies assumption ( $i$ i i $)$. Indeed, taking an arbitrary nonnegative function $x \in C(I)$ and $t_{1}, t_{2} \in I$ such that $t_{1} \leq t_{2}$, we obtain

$$
\begin{aligned}
\mid(H x)\left(t_{2}\right)- & (H x)\left(t_{1}\right) \mid-\left[(H x)\left(t_{2}\right)-(H x)\left(t_{1}\right)\right] \\
= & \left|h\left(t_{2}, x\left(t_{2}\right)\right)-h\left(t_{1}, x\left(t_{1}\right)\right)\right|-\left[h\left(t_{2}, x\left(t_{2}\right)\right)-h\left(t_{1}, x\left(t_{1}\right)\right)\right] \\
= & \left|\frac{2 t_{2} x\left(t_{2}\right)}{5\left(1+t_{2}\right)}-\frac{2 t_{1} x\left(t_{1}\right)}{5\left(1+t_{1}\right)}\right|-\left[\frac{2 t_{2} x\left(t_{2}\right)}{5\left(1+t_{2}\right)}-\frac{2 t_{1} x\left(t_{1}\right)}{5\left(1+t_{1}\right)}\right] \\
\leq & \left|\frac{2 t_{2} x\left(t_{2}\right)}{5\left(1+t_{2}\right)}-\frac{2 t_{2} x\left(t_{1}\right)}{5\left(1+t_{2}\right)}\right|+\left|\frac{2 t_{2} x\left(t_{1}\right)}{5\left(1+t_{2}\right)}-\frac{2 t_{1} x\left(t_{1}\right)}{5\left(1+t_{1}\right)}\right| \\
& -\left[\frac{2 t_{2} x\left(t_{2}\right)}{5\left(1+t_{2}\right)}-\frac{2 t_{2} x\left(t_{1}\right)}{5\left(1+t_{2}\right)}+\frac{2 t_{2} x\left(t_{1}\right)}{5\left(1+t_{2}\right)}-\frac{2 t_{1} x\left(t_{1}\right)}{5\left(1+t_{1}\right)}\right] \\
\leq & \frac{2 t_{2}}{5\left(1+t_{2}\right)}\left|x\left(t_{2}\right)-x\left(t_{1}\right)\right|+\left|\frac{2 t_{2}}{5\left(1+t_{2}\right)}-\frac{2 t_{1}}{5\left(1+t_{1}\right)}\right| x\left(t_{1}\right) \\
& -\frac{2 t_{2}}{5\left(1+t_{2}\right)}\left[x\left(t_{2}\right)-x\left(t_{1}\right)\right]-\left[\frac{2 t_{2}}{5\left(1+t_{2}\right)}-\frac{2 t_{1}}{5\left(1+t_{1}\right)}\right] x\left(t_{1}\right) \\
\leq & \frac{2 t_{2}}{5\left(1+t_{2}\right)}\left\{\left|x\left(t_{2}\right)-x\left(t_{1}\right)\right|-\left[x\left(t_{2}\right)-x\left(t_{1}\right)\right]\right\} \leq \frac{2 t_{2}}{5\left(1+t_{2}\right)} i(x) \leq \frac{1}{5} i(x) .
\end{aligned}
$$


In this case the first inequality (4.2) of assumption (vii) has the form

$$
\frac{1}{5} \Gamma\left(\frac{3}{2}\right) \Gamma\left(\frac{4}{3}\right)+\frac{1}{30} r^{3} \leq \Gamma\left(\frac{3}{2}\right) \Gamma\left(\frac{4}{3}\right) r,
$$

and this admits $r_{0}=1$ as a positive solution. Moreover,

$$
c\left\|k_{1}\right\|\left\|k_{2}\right\| r_{0}^{2}=\frac{1}{5} \frac{1}{2} \frac{1}{3} .1<\Gamma\left(\frac{3}{2}\right) \Gamma\left(\frac{4}{3}\right)=\frac{1}{2} \Gamma\left(\frac{1}{2}\right) \frac{1}{3} \Gamma\left(\frac{1}{3}\right) .
$$

On the other hand, we have $\Gamma\left(\frac{1}{2}\right)=1.77245$ and $\Gamma\left(\frac{1}{3}\right)=2.67893$. Then Theorem 8 guarantees that equation (4.7) has a nondecreasing solution.

Corollary 1. Let the assumptions of Theorem 8 be satisfied (with $m=n=1$ ), then the fractional-order quadratic integral equation

$$
\begin{aligned}
x(t)=a(t)+h(t, x(t)) \int_{0}^{t} \frac{(t-s)^{\alpha-1}}{\Gamma(\alpha)} k_{1}( & f(t, s)) x(s) d s \\
& \times \int_{0}^{t} \frac{(t-s)^{\beta-1}}{\Gamma(\beta)} k_{2}(g(t, s)) x(s) d s,
\end{aligned}
$$

has at least one solution $x \in C(I)$.

Corollary 2. Let the assumptions of Theorem 8 be satisfied (with $h(t, x(t))=1$ ), then the fractional-order quadratic integral equation

$$
x(t)=a(t)+\int_{0}^{t} \frac{(t-s)^{\alpha-1}}{\Gamma(\alpha)} k_{1}(f(t, s)) x(s) d s . \int_{0}^{t} \frac{(t-s)^{\beta-1}}{\Gamma(\beta)} k_{2}(g(t, s)) x(s) d s,
$$

has at least one solution $x \in C(I)$.

Corollary 3. Let the assumptions of Theorem 8 be satisfied ( with $k_{1}=k_{2}=I, \alpha=$ $\beta, f=g, a(t)=0)$, then the fractional-order quadratic integral equation

$$
x(t)=\left[\int_{0}^{t} \frac{(t-s)^{\alpha-1}}{\Gamma(\alpha)} f(t, s) x(s) d s\right]^{2},
$$

has at least one solution $x \in C(I)$.

\section{REFERENCES}

[1] R. P. Agarwal, M. Benchohra, and D. Seba, "On the application of measure of noncompactness to the existence of solutions for fractional differential equations," Results Math., vol. 55, pp. 221230, 2009.

[2] R. P. Agarwal and D. Oregan, "Singular volterra integral equations," Appl. Math. Lett., vol. 33, pp. 115-120, 2000.

[3] R. P. Agarwal and D. O'Regan, Fixed point theory and applications. Cambridge university press, 2004.

[4] A. Aghajani, J. Banaś, and Y. Jalilian, "Existence of solution for a class of nonlinear voltrra sigular integral equations," Computer and Mathematics with Applications, vol. 62, pp. 1215-1227, 2011.

[5] A. Aghajani, J. Banaś, and N. Sabzali, "Some generalizations of darbo fixed point theorem and applications," Bull. Belg. Math.Soc. Simon Stevin, vol. 20, pp. 345-358, 2013. 
[6] A. Aghajani and N. Sabzali, "A coupled fixed point theorem for condensing operators with application to system of integral equations," J. Nonlinear Convex Anal., vol. 15, pp. 941-952, 2014.

[7] J. Appell and P. P. Zabrejko, Nonlinear Superposition Operators. Cambridge university press, 1990.

[8] J. Banaś, "Measures of noncompactness in the space of continuous tempered functions," Demonstratio Math., vol. 14, pp. 127-133, 1981.

[9] J. Banaś and K. Goebel, "Measures of noncompactness related to monotonicity," Lecture Notes in Pure and Applied Mathematics, Marcel Dekker, Inc, vol. 60, 1980.

[10] J. Banaś and L. Olszowy, "Measures of noncompactness related to monotonicity," Comment. Math., vol. 41, pp. 13-23, 2001.

[11] W. G. El Sayed and B. Rzepka, "Nondecreasing solutions of a quadratic integral equation of urysohn type," Comput .Math. Appl., vol. 51, pp. 1065-1074, 2006.

[12] X. Hu and J. Yan, "The global attractivity and asymptotic stability of solution of a nonlinear integral equation," J. Math. Anal. Appl., vol. 321, pp. 147-156, 2006.

[13] L. Liu, F. Guo, and Y. Wu, "Existence theorems of global solutions for nonlinear volterra type integral equations in banach spaces," J. Math. Anal. Appl., pp. 638-649, 2005.

[14] Z. Liu and A. Kang, "Existence and asymptotic stability of solutions to functional-integral equation," Taiwan J. Math., vol. 11, pp. 187-196, 2007.

[15] M. Mursaleen and A. Alotaibi, "Infinite system of differential equations in some spaces," Abstr. Appl. Anal., 2012.

[16] M. Mursaleen and S. Mohiuddine, "Applications of measures of noncompactness to the infinite system of differential equations in $l_{p}$ spaces," Nonlinear Anal., Theory Methods Appl., vol. 75, pp. 2111-2115, 2012.

[17] B. Samet, C. Vetro, and F. Vetro, "From metric spaces to partial metric spaces," Fixed Point Theory Appl., 2013.

Author's address

R. Arab

Department of Mathematics, Sari Branch, Islamic Azad University, Sari, Iran

E-mail address: mathreza.arabeiausari.ac.ir 